\title{
Evaluación de la alfabetización digital y pedagógica en TIC, a partir de las opiniones de estudiantes en Formación Inicial Docente'
}

\author{
Pedro Sandoval Rubilar" \\ Francisco Rodriguez Alveal" \\ Ana Carolina Maldonado Fuentes"
}

I- Investigación financiada por el proyecto Formación Inicial Docente: niveles de competencia, conocimiento y uso profesional de las TIC que poseen estudiantes de ingreso y término en la formación inicial docente: un estudio comparativo. Universidad del Bío-Bío, Chile. Código. DIUBB 124823 3/R.

II- Universidad del Bío-Bío, Chillán, Chile. Contactos: psandoval@ubiobio.cl

frodriguez@ubiobio.cl

amaldonado@ubiobio.cl

\section{Resumen}

El artículo presenta los resultados sobre acceso, conocimiento y usos de herramientas TIC declarados por estudiantes de Pedagogía, pertenecientes a una institución de Educación Superior de Chile. Se pretende responder a la interrogante sobre el tipo de alfabetización que poseen y el que les deberían brindar su proceso de formación para su futuro ejercicio como profesores. El objetivo del estudio es diagnosticar las necesidades de formación en TIC de los futuros profesores del sistema escolar. Metodológicamente, es un estudio cuantitativo descriptivo transversal, con una población de 127 estudiantes de la cohorte 2014, pertenecientes a cuatro carreras de Pedagogía; se aplicó un cuestionario debidamente validado (Alpha de Cronbach 0,89). En el análisis de la información se utilizaron métodos descriptivos. Se concluye que los estudiantes de Pedagogía poseen un alto acceso a los recursos TIC, y, por lo mismo, se puede asumir que son sujetos nativos con grados de alfabetización digital suficiente que les permite acceder y usufructuar de dichos recursos sin dificultades. Sin embargo, se devela que no conocen ni usan programas específicos para el campo de la educación y tampoco saben cómo este dominio que poseen se puede traducir en su futuro desempeño profesional, especialmente a nivel aula.

\section{Palabras clave}

Formación inicial docente - Currículo - TIC - Evaluación. 


\title{
Evaluation of digital and pedagogical literacy in ICT based on the opinions of Initial Teacher Education students'
}

\author{
Pedro Sandoval Rubilar" \\ Francisco Rodriguez Alveal" \\ Ana Carolina Maldonado Fuentes"
}

\begin{abstract}
This paper presents the results about access, knowledge and uses of ICT tools declared by teacher students from a higher education institution in Chile. The objective is to examine the kind of literacy that they have and the one that should be provided by the training process for their future practice as teachers. This study aims to diagnose the needs of ICT training of future teachers of the Chilean school system. From the methodological point of view, this is a descriptive quantitative study, conducted with 127 students in the 2014 cohort, of four teaching programs, to which a validated questionnaire was applied (Cronbach Alpha 0.89). Descriptive methods were used for the analysis of information. In conclusion, students of the teaching programs have high access to ICT resources and, therefore, it is assumed that they are native individuals with sufficient digital literacy that allows them to have access and benefit from such resources without difficulties. However, they do not know or use specific programs for the field of education, and they do not know how their current mastery can be translated into their future professional performance either, especially in the classroom.
\end{abstract}

\section{Keywords}

Initial teacher education - Curriculum - ICT - Evaluation.

I- Investigación financiada por el proyecto Formación Inicial Docente: niveles de competencia, conocimiento y uso profesional de las TIC que poseen estudiantes de ingreso y término en la formación inicial docente: un estudio comparativo. Universidad del Bío-Bío, Chile. Código. DIUBB 124823 3/R.

II- Universidad del Bío-Bío, Chillán, Chile. Contacts: psandoval@ubiobio.cl

frodriguez@ubiobio.cl

amaldonado@ubiobio.cl 


\section{Antecedentes}

Actualmente existe un amplio consenso que las TIC han generado un cambio en la sociedad de la información y en la forma de relacionarse entre los individuos, por ello no ha de extrañar la terminología de sujetos nativos o alfabetización en la TIC como nuevas categorías en estudios y reflexiones recientes para referirse a este nuevo fenómeno de la sociedad actual (COLL, 2005). Por lo mismo, no es de extrañar la necesidad de integrar este nuevo fenómeno a los procesos formales de educación inicial, primaria, secundaria y terciaria (SANDOVAL; RODRÍGUEZ; MALDONADO, 2011).

En este contexto, desde 1992 el Ministerio de Educación de Chile (MINEDUC), implementa un proyecto denominado Enlaces, con el objetivo de proporcionar a los establecimientos educacionales computadores, infraestructura de redes de comunicación, internet e intranet en las escuelas, y de esta forma ir incorporando las nuevas tecnologías de información y comunicación a la educación (CHILE, 2012).

Posteriormente, el Ministerio de Educación impulsa estándares TIC para la formación inicial docente con el propósito de que este nuevo fenómeno sea asumido en las aulas por los profesores con la finalidad de favorecer habilidades digitales en los futuros ciudadanos $y$, por otra parte, integrar dichas herramientas a los procesos de enseñanza y aprendizaje para favorecer el logro de los aprendizajes esperados por el Currículo Nacional (VAILLANT, 2013).

No conforme con lo anterior, y para resaltar la importancia de dicha política, el propio Ministerio, impulsa una evaluación nacional a todos los egresados de Pedagogía, de las diferentes instituciones formadoras, con el objetivo de recolectar información sobre el nivel o dominio de competencias que tienen para ejercer la profesión; en este contexto, para el Ministerio de Educación de Chile, los futuros docentes no sólo deben dominar aspectos referidos a los conocimientos pedagógicos y disciplinares, sino también, los referidos a competencias TIC.
Asimismo,

[...] la incorporación de las TIC a la educación formal y escolar viene siendo justificada, reclamada o promovida, según los casos, desde hace ya varias décadas con el argumento de su potencial contribución a la mejora del aprendizaje y de la calidad de la enseñanza. Sin embargo, como muestra la literatura especializada, este argumento no ha encontrado hasta ahora un apoyo empírico suficiente. (COLL, 2004, p. 4).

Algunos estudios sugieren que "la escuela aún está lejos de superar la brecha digital que la separa de la evolución y desarrollo que la sociedad experimenta fuera de sus muros" (CUADRAD0, 2008, p. 2). Ello se puede deber a múltiples factores, desde los propios modelos de análisis de los estudios o la real posibilidad de que la TIC sean potenciales para generar cambios en la prácticas pedagógicas o didácticas (COLL, 2004).

Más allá de los enfoques teóricos en que se aborde la problemática de las reales posibilidades de impacto de las TIC en los procesos de enseñanza y aprendizaje, existe una tendencia a focalizar al profesor como sujeto clave de la discusión, ya sea como mediador de las prácticas socioculturales relacionadas con las TIC o como meros planificadores de recursos didácticos TIC (asumiendo que son neutros socialmente) como herramientas al servicio de la eficacia del proceso enseñanza aprendizaje (COLL, 2004; PEIRANO; DOMÍNGUEZ, 2008; TELLO; AGUADED, 2009; SANDOVAL, RODRÍGUEZ; MALDONADO, 2011).

En consecuencia, es posible profundizar en el análisis desde una doble cuestión. Por un lado, los sujetos en formación inicial docente están inmersos en una sociedad de las tecnologías y la comunicación (producto de una sociedad del conocimiento y comunicación que ha cambiado al mundo y sus sujetos), sean nativos digitales o no. Por otro, estos sujetos deben responder a la demanda de emplear las TIC en el campo educativo en su futuro 
desempeño profesional. En otras palabras, la problemática del estudio pretende resolver qué tan nativos son estos sujetos y si ello les permite estar mejor preparados para trabajar con las TIC en su futuro desempeño profesional en el aula. Dicho lo anterior, el presente estudio pretende reflexionar en torno a esta problemática, recolectando información desde los mismos actores sobre al acceso, uso, conocimiento de las TIC y acceso, conocimiento y uso pedagógico de las TIC para su futuro profesional.

\section{Problema}

La incorporación de las TIC en la formación inicial docente es demandada por una doble necesidad. En primer lugar, para generar procesos pertinentes de socialización y endoculturización de las nuevas generaciones, lo que supone que la escuela y los docentes deben asumir dicho fenómeno como parte central de sus prácticas de transmisión socio-cultural. En segundo lugar, las TIC al ser consideradas como herramientas potenciales de mejoramiento de la calidad de la educación, son recursos curriculares y didácticos relevantes para mejorar los aprendizajes y por ende, los indicadores de calidad de la educación (SANDOVAL; RODRÍGUEZ; MALDONADO, 2011).

Resulta evidente, según la literatura especializada, que las generaciones actuales son sujetos nativos de estas nuevas tecnologías. Sin embargo, queda por explorar en profundidad si, además, como estudiantes en formación docente y futuros profesores, son igualmente alfabetizados en el campo de la pedagogía.

$\mathrm{Si}$ bien este problema puede resultar conocido, generalmente, tiende a responderse desde la reflexión teórica (deber ser) o asumiendo ciertos supuestos sobre el dominio y conocimiento de las tecnologías que poseen los estudiantes (alfabetización), con escasa información desde los propios actores. En otras palabras, si los actuales estudiantes son nativos de estas tecnologías, lo más probable es que deberían poseer algún grado de alfabetización digital. En este contexto, cualquier respuesta sobre su trayectoria formativa y en qué debe sustentarse el desarrollo de las competencias TIC, debería tener como punto de inicio su propia alfabetización digital.

Otro punto problemático, dice relación en qué medida es posible relacionar el grado de alfabetización que poseen los estudiantes por ser nativos en las TIC y el empleo de las mismas en su futuro profesional, es decir, determinar si la alfabetización digital de los estudiantes como nativos de una sociedad basada en las nuevas TIC es útil o suficiente para ser alfabetizados pedagógicamente; es decir, para el uso y empleo de estas TIC en su quehacer pedagógico y profesional.

Dicho lo anterior, en otras palabras, el problema radica en si es posible transferir el nivel de alfabetización entre prácticas de distintas naturalezas. Por ello, el presente estudio pretende explorar las opiniones de los propios destinatarios sobre su conocimiento y dominio de las TIC, teniendo como referencia estos dos tipos de alfabetización. Las preguntas que orientarán el estudio son: ¿qué tan familiarizados están los estudiantes de la formación inicial docente con las nuevas tecnologías y herramientas informáticas según su propia opinión?, ¿cuál es el nivel de acceso y uso que hacen de las TIC?, y finalmente, ¿qué tan preparados están para usar las TIC como recurso de enseñanza y aprendizaje en su futuro desempeño profesional?

\section{Objetivos del estudio}

\section{Objetivo general}

Diagnosticar el tipo de alfabetización en TIC que poseen los futuros profesores del sistema escolar chileno, a partir de las opiniones de los estudiantes en formación inicial docente, en una universidad del Consejo de Rectores del centro sur de Chile.

\section{Objetivos específicos}

1. Caracterizar el acceso y uso de las TIC que tienen los estudiantes de Pedagogía. 
2. Describir el tipo de uso que hacen de las TIC los estudiantes de Pedagogía.

3. Identificar las fortalezas y debilidades que tienen los estudiantes de Pedagogía, desde sus propias opiniones, para el uso pedagógico de las TIC en su futuro profesional.

\section{Marco operativo}

\section{Alfabetización digital}

Inicialmente la alfabetización se relacionaba con la lectura y escritura, entendiéndose como un estado que una vez logrado se poseía permanentemente. Sin embargo, ambas ideas iniciales asociadas al concepto de alfabetización han mutado para entenderlas como un concepto complejo y dinámico. Así pues, Caviglia acuña el concepto de alfabetización avanzada para referirse a procesos relacionados y distintos a la lectura y escriturar (CAVIGLIA, 2003 apud RODRÍGUEZ, 2004).

Según Rodríguez (2004) las nuevas tendencias permiten comprender la alfabetización como un concepto plural, procesual y no limitado al lenguaje oral y escrito, sino a las manifestaciones comunicativas de cualquier índole cultural. Así pues, este autor plantea que la "la alfabetización puede ser pensada como una práctica, es decir como una actividad del sujeto que es significativa y que, además, transforma la realidad” (RODRÍGUEZ, 2004, p. 4).

En esa línea, Scribner y Cole ya en 1981 sostenían que la alfabetización es

[...] un conjunto de prácticas socialmente organizadas que hace uso de un sistema de símbolos y de una tecnología para producirlo y diseminarlo. La alfabetización no es simplemente saber cómo leer y escribir un texto determinado sino la aplicación de este conocimiento para propósitos específicos en contextos específicos. La naturaleza de esas prácticas incluyendo, desde luego, sus aspectos tecnológicos determinarán los tipos de habilidades asociadas con la alfabetización (SCRIBNER; COLE, 1981 apud RODRÍGUEZ, 2004, p. 4-5).

Al ser la alfabetización una práctica social, se concibe como procesual y por ende

[...] lejos de acabarse en un momento dado, continúan evolucionando y cambiando en momentos posteriores (como, por ejemplo, los propios contenidos digitales que no paran de ser cambiantes, o los propios de las distintas funcionalidades que pueden ser diferentes para cada sujeto); es, si se quiere, un proceso abierto con re-alfabetizaciones... El carácter gradual de la alfabetización es reconocer que se trata de un continuo, si se quiere de tipo competencial, en el que hay muchas posiciones y no sólo dos categorías (alfabetizado/analfabeto) (SCRIBNER; COLE, 1981 apud RODRÍGUEZ, 2004, p. 3-4).

Por otro lado, comprender la alfabetización como práctica, es incorporar la dimensión contextual, social y simbólica de la cultura en dichas prácticas de alfabetización. Así pues, "cambia el foco de análisis: dejando de preocuparse únicamente por los resultados, $\mathrm{y}$ mostrando la relación entre el contexto cultural (y tecnológico) y las formas de utilización concreta por los sujetos" (p. 4).

En consecuencia, en este nuevo contexto de alfabetización tecnológica, comprender dicho proceso de forma estática y meramente funcional (aprender habilidades, técnicas y procedimientos de determinados programas o aplicaciones), sin sus referencias sociales, culturales y comunicativas e intencionales resulta una mirada estrecha.

En este sentido, es posible establecer los siguientes niveles de alfabetización en el marco de las TIC:

a) unos claramente tecnológicos, relativos a la materialidad que soporta las prácticas alfabetizadas; b) otros relacionados 
con las competencias lingüísticas $\mathrm{y}$ extralingüísticas, 0 , en general, con las capacidades cognitivas asociadas a su uso; $\mathrm{c}$ ) otros relativos a las prácticas, a su contexto, a cómo son usadas y a las consecuencias sociales y personales de esa utilización; d) otros, en fin, a la capacidad crítica para pensar las prácticas digitales como prácticas socialmente construidas. (p. 10).

De lo anterior se desprende que hay diferentes niveles de alfabetización de las TIC en la sociedad actual. Por lo mismo, considerando que los estudiantes de pedagogía están sometidos a prácticas propias de las tecnologías de la comunicación modernas, cabe preguntarse si el nivel de alfabetización desarrollado por ellos en sus prácticas cotidianas con las TIC se relaciona con el desarrollo de una alfabetización pedagógica. En especial, esto cobra relevancia, pues supone abordar las TIC desde otras prácticas propias del campo educativo, con un contexto social y cultural de usos particulares.

\section{Alfabetización digital y Alfabetización pedagógica}

En el punto anterior, se desprende que las

[...] alfabetizaciones digitales nos muestran como uno de los grandes problemas educativos actuales: en un mundo cada vez más digitalizado, la idea misma de ser competente en las nuevas prácticas letradas está sometida a la tensión entre los nuevos medios y sus nuevas maneras de significar y comunicar y, por otra parte, a unas prácticas educativas muchas veces todavía pensadas para una sociedad que ya se ha transformado profundamente (p. 17).

De lo anterior se desprende que, el hecho que un sujeto se encuentre alfabetizado digitalmente para acceder, transmitir y compartir información mediante el uso de nuevas tecnologías, de manera alguna implica que esté alfabetizado para enseñar, acceder y producir conocimiento en el aula, procesos vinculados a la naturaleza propia de su quehacer como profesor. Tal como lo señala Coll (2004), manejar información no

[...] es sinónimo de conocimiento ni la recepción o el acceso a la información garantiza el aprendizaje. La información se convierte en conocimiento y el acceso a la información da lugar al aprendizaje cuando actuamos sobre ella, la procesamos, la organizamos, nos la apropiamos, la utilizamos y la confrontamos con otros; en suma, cuando somos capaces de darle significado y sentido (COLL, 2004, p. 4).

Por tanto, la alfabetización pedagógica de las TIC se relaciona con prácticas de procesar y organizar didácticamente dichos recursos, además de dotar dichas prácticas con un sentido y significado cultural, asunto que en un contexto escolar se relaciona con el currículo como proyecto cultural para las nuevas generaciones. No basta con usar los recursos TIC o acceder a la información y comunicación mediante su empleo para enseñar, pedagógicamente se trata de acceder a los códigos culturales de los saberes expresados en el currículo escolar mediante prácticas didácticas con TIC.

En ese contexto, el presente estudio asume que las prácticas pedagógicas son aquellas que se realizan en el aula, en un contexto social especifico, en función de un marco curricular escolar, donde se definen códigos, metas, aprendizajes, contenidos y medios (entre ellos los tecnológicos o TIC) para lograr un aprendizaje, una habilidad o conocimiento por parte del educando ${ }^{1}$.

Dicho lo anterior, el presente estudio asume el supuesto que los estudiantes actuales de pedagogía son nativos de las TIC y, por ello, poseen un nivel de alfabetización tecnológica según lo definido por Rodríguez (2004). Es decir, cuentan con un cierto nivel en el conocimiento y

1- Dicha conceptualización es una adaptación del planteamiento de Mónica Isabel Perazzo (2008). 
usos de dichas herramientas, las que constituyen la materialidad que permite todas las demás prácticas y niveles de alfabetización. No obstante, queda por resolver si este nivel y práctica de alfabetización será suficiente para transferir el mismo nivel de alfabetización a las prácticas pedagógicas tal como se definió anteriormente.

\section{Diseño metodológico}

\section{Selección y composición de la muestra}

Para el cálculo de la muestra se realizó un muestreo aleatorio simple considerando un error de un 5\% aproximadamente, un nivel de confianza del 95\% y un factor de corrección por población finita, obteniendo un tamaño muestral mínimo de 123 estudiantes, por lo cual, para efectos del estudio se consideraron 127 casos. Todos pertenecen a carreras de Pedagogía de la cohorte 2014, de una universidad regional del Consejo de Rectores del centro sur de Chile, de carácter público, de ambos géneros (57,5\% femenino y 42,5\% masculino) cuya edad fluctúa entre 17 y 39 años con un promedio de 18,7 años (Desviación Estándar - D.E. 4,6 años). Los estudiantes en su mayoría presentan residencia urbana $(75,6 \%)$ y rural $(19,7 \%)$. En relación a la dependencia del colegio de procedencia se tiene que mayoritariamente provienen de colegios particulares subvencionados (66,9\%), y en menor proporción de establecimientos de dependencia municipal (31,5\%), siendo solamente un $0,8 \%$ de colegios particulares pagados.

En la siguiente tabla, se muestra la distribución por quintiles de los estudiantes de las carreras bajo estudio, dado que son una forma de clasificar a la población chilena según su nivel de ingresos. Mayoritariamente se ubican entre el primer y tercer quintil, es decir, con ingresos per cápita familiar de a los más US\$ 307,49. Solamente estudiantes de la carrera de Pedagogía en Historia y Geografía se ubican dentro del cuarto y quinto quintil, con porcentajes del 10,53\% y 5,26\% respectivamente. Una proporción menor de estudiantes de Pedagogía en Educación Matemática se ubica en el cuarto quintil $(6,52 \%)$, porcentaje que disminuye a un 3,03\% en los estudiantes de Pedagogía en Educación General Básica.

Tabla 1- Distribución porcentual de la cohorte 2014 según carrera en relación a los quintiles en dólares.

\begin{tabular}{|c|c|c|c|c|c|}
\hline Carrera & $\begin{array}{l}\text { Quintil I } \\
0 \text { a } 119,38\end{array}$ & $\begin{array}{c}\text { Quintil II } \\
119,38 \text { a 199,94 }\end{array}$ & $\begin{array}{c}\text { Quintil III } \\
199,94 \text { a } 307,50\end{array}$ & $\begin{array}{c}\text { Quintil IV } \\
307,50 \text { a } 561,70\end{array}$ & $\begin{array}{c}\text { Quintil V } \\
561,70 \text { y más. }\end{array}$ \\
\hline Educación General Básica & $48,48 \%$ & $21,21 \%$ & $15,15 \%$ & $3,03 \%$ & $0,00 \%$ \\
\hline Pedagogía en Educación Matemática & $47,83 \%$ & $23,91 \%$ & $10,87 \%$ & $6,52 \%$ & $0,00 \%$ \\
\hline Pedagogía en Ciencias Naturales & $46,67 \%$ & $33,33 \%$ & $13,33 \%$ & $0.00 \%$ & 0.00 \\
\hline Pedagogía en Historia y Geografía & $35,09 \%$ & $35,09 \%$ & $12,28 \%$ & $10,53 \%$ & $5,26 \%$ \\
\hline
\end{tabular}

Fuente: Banco Central de Chile, dólar observado \$ 590,91 - 01 sept. 2014

En resumen, de los datos se puede desprender que los estudiantes de la cohorte 2014 de las carreras bajo estudio pertenecen a una población vulnerable económicamente.

\section{Tipo de estudio y análisis de datos}

Se trata de un estudio descriptivo de corte transversal (COOK; REICHARDT, 1986;
LATORRE; DEL RINCÓN; ARNAL, 1997), realizado a estudiantes de Pedagogía de una Facultad de Educación en una universidad del Consejo de Rectores, durante el primer semestre de 2014. En el análisis de la información se utilizaron métodos descriptivos numéricos, promedio y porcentajes, coeficiente de correlación de Spearman considerando un nivel de significación del 5\%. El procesamiento de 
la información se realizó en el programa SPSS versión 13.0 para Windows.

\section{Instrumentos}

En la recolección de la información se utilizó un cuestionario de tipo encuesta, denominado Acceso, uso y percepción de necesidades de conocimiento de TIC para docentes en formación inicial, con 42 reactivos de distinto formato: algunas de las respuestas eran de tipo dicotómica, otras de selección de alternativas y un grupo de respuestas asociadas a una escala tipo Likert. El instrumento se elaboró adaptando la Encuesta sobre equipamiento y uso de tecnologías de información y comunicación en los hogares (TIC-H) 2005 de España (INE, 2005).

La estructura del instrumento se compone de cinco secciones: la primera es sobre el acceso a recursos TIC; la segunda, sobre el acceso a conectividad (internet); la tercera, sobre frecuencia de uso del computador; la cuarta, sobre el tipo y frecuencia de uso del computador e internet; y, finalmente, la quinta parte, sobre las necesidades de formación en TIC declaradas por los estudiantes para su futuro desempeño profesional.

Tabla 2- Tabla de especificaciones del instrumento: acceso, uso y percepción de necesidades de conocimiento de TIC para docentes en formación inicial.

\begin{tabular}{|c|c|c|c|}
\hline \multicolumn{2}{|r|}{ Categoría } & $\mathrm{N}^{\circ}$ Preguntas & TIPO DE ÍTEM \\
\hline Parte I & Acceso a recursos TIC. & 10 & Respuesta dicotómica \\
\hline Parte II & Acceso a conectividad a internet. & 4 & $\begin{array}{c}\text { Respuesta dicotómica y Selección de } \\
\text { alternativa }\end{array}$ \\
\hline Parte III & Frecuencia de uso del computador & 2 & Respuesta dicotómica \\
\hline Parte IV & Tipo y frecuencia de uso del computador e internet & 16 & Escala tipo Likert \\
\hline Parte V & $\begin{array}{l}\text { Necesidades de formación en TICs declaradas por los } \\
\text { estudiantes para su futuro ejercicio profesional. }\end{array}$ & 10 & Escala tipo Likert \\
\hline Total & & & 42 \\
\hline
\end{tabular}

Fuente: tabla elaborada por los autores para el presente estudio

El instrumento fue sometido a proceso de validación, obteniendo un Alpha de Cronbach 0,89 lo que implica que posee una fuerte consistencia interna; siendo además el índice de validez de contenido de 0,71 , lo cual nos evidencia que la información entregada por el instrumento es confiable para efectos del estudio.

\section{Resultados}

Los resultados obtenidos de la aplicación del instrumento se presentarán siguiendo el orden de los objetivos del estudio, a saber: caracterizar el acceso y uso de las TIC que tienen los estudiantes de Pedagogía; describir el tipo de uso que hacen de las TIC los estudiantes de Pedagogía e identificar las fortalezas y debilidades que tienen los estudiantes de pedagogía, desde sus propias opiniones, para el uso pedagógico de las TIC en su futuro profesional.

\section{Acceso y frecuencia de uso de recursos TIC}

\section{Acceso a recursos TIC}

En relación con la tenencia de tecnologías TIC, los resultados evidencian que un 99,2\% de los alumnos encuestados tienen teléfono móvil y el 100,0\% correo electrónico, como así también cuentan con algún dispositivo de almacenamiento externo como pendrives o disco duro externo. En la siguiente tabla se muestra la distribución porcentual según carreras de ingreso, en la cual podemos observar que el 65,4\% de alumnos encuestados 
cuentan con computador fijo o de escritorio. El porcentaje anterior se puede explicar debido a que los alumnos actualmente privilegian tener un computador portátil $(84,3 \%)$ pues lo pueden utilizar en cualquier parte, ya sea en: instancias formales de clase y/o en casa para realizar sus tareas o trabajos. No obstante lo anterior, la introducción de los tablet a nivel de los estudiantes es porcentualmente más baja, dado que solamente un 7,9\% dice contar con estos equipos (Tabla 3).

Un dato de interés sobre la muestra bajo estudio, es que aproximadamente un 50,4\% de los estudiantes tiene acceso a computadores e internet en sus hogares; al mismo tiempo, el $85,0 \%$ tiene acceso a internet y computadores portátiles en sus lugares de estudio. Cabe señalar que los estudiantes encuestados comparten en la sede una infraestructura institucional adaptada para la docencia, que incluye $3.060 \mathrm{~m}^{2}$ en laboratorios de especialidad computación y talleres, a lo que se añade la posibilidad de acceder a computadores portátiles que se pueden utilizar dentro del campus universitario, en coherencia con las políticas de la entidad.

Tabla 3- Distribución porcentual del acceso a TIC de los encuestados en el estudio.

\begin{tabular}{|c|c|c|}
\hline Recursos TIC. & Sí & No \\
\hline Tenencia de teléfono móvil & 99,2 & 0,8 \\
\hline ¿Tiene un computador fijo o de escritorio en casa?. & 65,4 & 34,6 \\
\hline ¿Tiene algún computador portátil (notebook o netbook) personal o familiar? & 84,3 & 15,7 \\
\hline ¿Tiene algún tablet personal o familiar? & 7,9 & 90,6 \\
\hline ¿Tiene mp3 o mp4? & 48,0 & 51,2 \\
\hline ¿Tiene acceso a computadores fijos su lugar de estudio? & 87,4 & 11,8 \\
\hline ¿Tiene acceso a computadores portátiles (notebook o netbook) en su lugar de estudio? & 95,3 & 3,9 \\
\hline ¿Tiene conexión a internet en casa?. & 67,7 & 31,5 \\
\hline ¿Tiene acceso a internet en su lugar de estudio? & 88,2 & 11,0 \\
\hline Computador fijo e internet en casa & 50,4 & 15,0 \\
\hline Computadores portátiles en su lugar de estudio con internet & 85,0 & 0,8 \\
\hline Acceso a notebook personal con internet en su hogar & 60,6 & 8,7 \\
\hline Acceso a notebook personal con acceso a internet en la universidad & 74,8 & 2,4 \\
\hline
\end{tabular}

Fuente: tabla elaborada por los autores para el presente estudio

Otro factor a tener en cuenta es la conectividad, en particular el acceso a internet desde los hogares. En este sentido, se observa que el $67,7 \%$ de estudiantes tiene acceso a internet desde sus casas, aumentando considerablemente el porcentaje $(85,0 \%)$ al consultarles si tienen acceso en su lugar de estudio. En buenas cuentas, es posible concluir que, en general, los estudiantes tienen conectividad y acceso a internet.

\section{Frecuencia de uso de TIC}

En cuanto al porcentaje de uso del computador fijo que poseen en sus hogares, el $37,0 \%$ de los estudiantes encuestados lo utiliza entre seis a siete días. En relación con la conexión a internet, un 55,9\% lo hace preferentemente desde sus hogares o lugar de residencia, cuyo porcentaje disminuye al 30,7\% en sus lugares de estudio, con valor de frecuentemente entre cuatro a cinco días de la semana. 
Tabla 4- Distribución porcentual de la frecuencia de uso de TIC de los estudiantes encuestados.

\begin{tabular}{|c|c|c|c|c|c|}
\hline & \multicolumn{5}{|c|}{ Frecuencia de uso semanal } \\
\hline & No Tengo/No lo Uso & Menos de 2 días & 2 a 3 días & 4 a 5 días & 6 a 7 días \\
\hline El computador fijo o de escritorio de mi casa lo uso & 22,0 & 13,4 & 9,4 & 14,2 & 37,0 \\
\hline $\begin{array}{c}\text { Me conecto a internet desde mi hogar o lugar de } \\
\text { residencia }\end{array}$ & 11,0 & 6,3 & 11,0 & 15,0 & 55,9 \\
\hline $\begin{array}{l}\text { Reviso el correo electrónico desde mi hogar o lugar } \\
\text { de residencia }\end{array}$ & 5,5 & 22,0 & 24,4 & 21,3 & 26,8 \\
\hline $\begin{array}{l}\text { El computador fijo o portátil al que tiene acceso en } \\
\text { su lugar de estudio lo uso }\end{array}$ & 5,5 & 23,6 & 24,4 & 19,7 & 26,8 \\
\hline Me conecto a internet desde mi lugar de estudio & 1,6 & 15,7 & 26,0 & 30,7 & 24,4 \\
\hline $\begin{array}{c}\text { Reviso el correo electrónico desde mi lugar de } \\
\text { estudio }\end{array}$ & 7,1 & 28,3 & 18,9 & 26,0 & 19,7 \\
\hline Accedo al correo o internet por mi teléfono móvil & 28,3 & 11,0 & 7,9 & 19,7 & 33,1 \\
\hline
\end{tabular}

Fuente: tabla elaborada por los autores para el presente estudio

Los resultados permiten afirmar que otro tema de interés para los estudiantes es la conectividad. En tal sentido, el 26,8\% de los encuestados revisan su correo electrónico desde su lugar de residencia entre seis y siete días; no obstante un 24,4\% lo hace entre dos y tres días a la semana. Lo anterior se puede deber a que, en general, los estudiantes prefieren otros medios de comunicación en tiempo real como por ejemplo whatsapp. Cabe hacer notar que el 33,1\% de los estudiantes accede al correo electrónico o internet desde su teléfono móvil entre seis y siete días a la semana, lo cual indicaría que, en general, los alumnos se mantienen conectados.

\section{Tipo de uso de los recursos TIC utilizados por los estudiantes de Pedagogía}

\section{Tipo de uso de internet}

Como se puede observar en la Tabla 5, las respuestas de los encuestados dan cuenta del tipo de uso que dan a internet, ya sea desde su domicilio o desde el lugar de estudio. En general, el 57,5\% de ellos la utiliza preferentemente para realizar trabajos académicos, cuyo porcentaje aumenta a un 83,5\% cuando se encuentra en su lugar de estudio; de manera similar, el 69,3\% de los estudiantes hace uso del correo electrónico para realizar trabajos académicos. Por el contrario, para comunicarse con familiares y amigos, su uso sería escaso, con porcentajes que varias de $0,0 \%$ a $9,4 \%$.

\section{Tipo de uso recursos TIC}

En general, el 55,9\% de los estudiantes utiliza los computadores fijos en su domicilio para realizar trabajos académicos, porcentaje que aumenta a un 82,7\% cuando cuentan con computadores fijos o portátiles en sus lugares de estudio. Lo anterior permite inferir que los estudiantes usan los computadores fijos o portátiles de preferencia para realizar trabajos académicos y los celulares para comunicarse entre ellos y su familia $(46,5 \%)$, lo que ciertamente se pude deber a las aplicaciones que actualmente traen consigo dichos aparatos, especialmente asociadas a redes sociales. 
Tabla 5- Distribución porcentual del tipo de uso de internet de los encuestados presentes en el estudio.

\begin{tabular}{|c|c|c|c|c|}
\hline & $\begin{array}{l}\text { Realizar trabajos } \\
\text { académicos }\end{array}$ & $\begin{array}{l}\text { Gestionar contactos y } \\
\text { trabajos }\end{array}$ & $\begin{array}{l}\text { Comunicarme con } \\
\text { familiares y amigos }\end{array}$ & $\begin{array}{l}\text { Ocio: lectura, } \\
\text { música, juegos }\end{array}$ \\
\hline $\begin{array}{l}\text { Me conecto a internet desde mi hogar o lugar de } \\
\text { residencia frecuentemente para }\end{array}$ & 57,5 & 26,0 & 3,9 & 3,1 \\
\hline El correo electrónico lo uso frecuentemente para & 69,3 & 20,5 & 6,3 & 0,8 \\
\hline $\begin{array}{l}\text { Me conecto a internet desde el lugar de estudio } \\
\text { frecuentemente para }\end{array}$ & 83,5 & 12,6 & 1,6 & 1,6 \\
\hline
\end{tabular}

Fuente: tabla elaborada por los autores para el presente estudio.

Tabla 6- Distribución porcentual del tipo de uso de TIC de los estudiantes encuestados.

\begin{tabular}{|c|c|c|c|c|}
\hline & $\begin{array}{c}\text { Realizar trabajos } \\
\text { académicos }\end{array}$ & $\begin{array}{c}\text { Gestionar contactos y } \\
\text { trabajos }\end{array}$ & $\begin{array}{l}\text { Comunicarme con } \\
\text { familiares y amigos }\end{array}$ & $\begin{array}{l}\text { Ocio: lectura, } \\
\text { música, juegos }\end{array}$ \\
\hline $\begin{array}{l}\text { El computador fijo o de escritorio de mi casa o lugar } \\
\text { de residencia lo uso frecuentemente para }\end{array}$ & 55,9 & 12,6 & 2,4 & 5,5 \\
\hline $\begin{array}{c}\text { El computador fijo o portátil del lugar de estudio lo } \\
\text { uso frecuentemente para }\end{array}$ & 82,7 & 10,2 & 1,6 & 3,1 \\
\hline Su teléfono móvil lo usa frecuentemente para & 6,3 & 37,8 & 46,5 & 6,3 \\
\hline
\end{tabular}

Fuente: tabla elaborada por los autores para el presente estudio

\section{Tipo de uso de herramientas (software) TIC}

En la siguiente tabla se aprecia el tipo de herramientas informáticas utilizadas por los encuestados en relación a la frecuencia de uso. Se observa que el 43,3\% de los estudiantes encuestados declara tener muy poco conocimiento acerca del uso de procesadores de texto y programas de presentaciones. Situación similar ocurre con lo relacionado a bases de datos, pues solo el 56,7\% expresa tener casi nada de conocimiento en relación a su uso, situación que puede ser catalogada como preocupante debido a que este tipo de software, como excel, está presente en los textos escolares entregados por el Ministerio de Educación chileno, como herramienta que permite visualizar fenómenos aleatorios o determinísticos.

Tabla 7- Distribución de los encuestados en relación al tipo de uso de herramientas informáticas en su rol de estudiantes.

\begin{tabular}{|c|c|c|c|c|c|}
\hline & Nula & Casi nada & Muy poco & Suficiente & Muchísimo \\
\hline Procesador de textos y programas de presentaciones. (word, powerpoint, etc.) & 0,8 & 20,5 & 43,3 & 33,1 & 2,4 \\
\hline Bases de datos, hojas de cálculo, (excel, etc.) & 24,4 & 56,7 & 15,0 & 2,4 & 1,6 \\
\hline Diseño de páginas web y materiales multimedia & 63,8 & 26,8 & 2,4 & 3,9 & 2,4 \\
\hline Programa de presentaciones avanzado (acromedia flash) & 3,1 & 33,9 & 35,4 & 25,2 & 2,4 \\
\hline Programas de comunicación (correo electrónico, foros, chats, etc.) & 3,9 & 22,8 & 14,2 & 26,8 & 31,5 \\
\hline Programas de software libre (programas tipo linux y otros) & 46,5 & 20,5 & 11,0 & 11,0 & 11,0 \\
\hline Programas de ocio: música, juegos, etc & 11,0 & 28,3 & 15,7 & 26,0 & 18,9 \\
\hline
\end{tabular}

Fuente: tabla elaborada por los autores para el presente estudio 
A nivel de enseñanza, los futuros docentes deben desarrollar actividades tendientes a la generación de páginas web o materiales multimediales, coherentemente con los actuales Estándares Orientadores (CHILE, 2011). En tal sentido, el 63,8\% de los encuestados presenta un conocimiento nulo al respecto y solamente un 2,4\% declara conocer muchísimo del tema. Situación similar ocurre con programas de presentación a nivel avanzado como macromedia flash, pues el 33,9\% presenta casi nada de conocimiento. No obstante lo anterior, cabe resaltar que solamente un $31,5 \%$ de los estudiantes presenta muchísimo conocimiento en relación al uso de programas de comunicación y un porcentaje no menor $(22,8 \%)$ presente casi nada de conocimiento al respecto. En relación al uso o manejo de programas de fuente libre o código abierto, el 46,5\% de los estudiantes presenta un conocimiento nulo y solamente un 11,0\% declara muchísimo conocimiento.

Por otro lado, se observa que, en general, los estudiantes no hacen uso de software de carácter más específico para su formación pedagógica; como por ejemplo, programas para planificación, evaluación, plataformas virtuales o pizarras digitales.

Tabla 8- Distribución de los encuestados en relación al uso de software relacionado con su futuro profesional

\begin{tabular}{|c|c|c|c|c|c|}
\hline & Nula & Casi nada & Muy poco & Suficiente & Muchísimo \\
\hline $\begin{array}{c}\text { Programas específicos de su campo profesional (planificación, } \\
\text { evaluación, etc.) }\end{array}$ & 24,4 & 38,6 & 23,6 & 11,8 & 0,0 \\
\hline $\begin{array}{l}\text { Plataformas virtuales para formarse (plataformas de enseñanza: } \\
\text { campus virtual, moodle) }\end{array}$ & 51,2 & 33,9 & 11,8 & 2,4 & 0,0 \\
\hline Pizarra electrónica o digital & 75,6 & 17,3 & 4,7 & 16,6 & 0,0 \\
\hline
\end{tabular}

Fuente: tabla elaborada por los autores para el presente estudio

En este sentido, el $38,6 \%$ de los estudiantes declara conocer casi nada de programas específicos de su campo profesional (relacionados con planificación y evaluación); de manera similar, el 33,9\% conoce casi nada de las plataformas virtuales, tales como campus virtual, moodle, etc. Destaca que un $75,6 \%$ de los estudiantes encuestados no tienen conocimientos del uso de la pizarra digital, un recurso presente en el sistema escolar chileno.

\section{Necesidades de formación TIC para uso pedagógico como futuro profesional}

\section{Dominio de herramientas TIC a nivel general}

A partir de la opinión de los estudiantes, se infiere el dominio que poseen de programas y aplicaciones, utilizando una escala tipo Lickert. En la Tabla 9 se muestra que el 80,3\% de los estudiantes, poseen muy poco dominio en programas como procesador de textos (word) y programa de presentación power point, $y$, asimismo, un 59,1\% un dominio nulo en programas como base de datos (excel). Así, se puede inferir que los estudiantes encuestados presentan muy poco o casi nada de dominio en programas de la microsoft offıce. Situación similar ocurre con el diseño de páginas web, dado que el 72,4\% declara tener casi nada de dominio en ese campo y en programas de presentación solamente un 9,4\% declara un dominio suficiente. El porcentaje aumenta a un 25,2\% al tratarse de programas de comunicación, de manera similar el 20,5\% de los estudiantes encuestados presenta un dominio suficiente en lo que dice relación a programas de ocio, etc. 
Tabla 9- Distribución porcentual de dominio de herramientas TIC de los estudiantes encuestados.

\begin{tabular}{|c|c|c|c|c|c|}
\hline & Nula & Casi nada & Muy poco & Suficiente & Muchísimo \\
\hline Procesador de textos y programas de presentaciones. (word, powerpoint, etc.) & 0,8 & 10,2 & 80,3 & 8,7 & 0,0 \\
\hline Bases de datos, hojas de cálculo, (excel, etc.) & 59,1 & 37,0 & 3,1 & 0,0 & 0,0 \\
\hline Diseño de páginas web y materiales multimedia & 0,0 & 72,4 & 22,0 & 3,1 & 0,0 \\
\hline Programa de presentaciones avanzado (macromedia flash) & 0,0 & 14,2 & 75,6 & 9,4 & 0,0 \\
\hline Programas de comunicación (correo electrónico, foros, chats, etc.) & 0,0 & 12,6 & 60,6 & 25,2 & 0,0 \\
\hline Programas de software libre (programas tipo linux y otros) & 0,0 & 49,6 & 34,6 & 13,4 & 0,0 \\
\hline Programas de ocio: música, juegos, etc & 0,0 & 26,8 & 51,2 & 20,5 & 0,0 \\
\hline
\end{tabular}

Fuente: tabla elaborada por los autores para el presente estudio

Como es sabido, el uso de software de fuente libre ha ido en aumento en las últimas décadas (RIOSECO, 2008) generándose comunidades que potencian el uso de este tipo de software. Cabe señalar que solamente un $13,4 \%$ de los estudiantes declaran un dominio del tipo suficiente al respecto.

\section{Dominio de herramientas TIC asociadas a su futuro profesional}

Por otro lado, en relación a programas específicos del futuro quehacer pedagógico, tales como software de planificación, evaluación, plataformas virtuales y pizarra digital, los estudiantes, en general, perciben que no cuentan $o$ no han desarrollado habilidades en ellas (52,8\%), en particular la pizarra digital declarando un dominio nulo o casi nulo $(83,5 \%)$, situación similar ocurre con plataformas virtuales donde el 59,1\% declara tener muy poco dominio.

En la siguiente tabla se muestra el coeficiente de correlación de Spearman, en ella se observa que existe una correlación significativa entre la frecuencia de uso de los programas específicos de su campo profesional con su percepción de dominio $(r=0,68, p$-value $<0,05)$.

Tabla 10- Distribución porcentual de dominio de herramientas TIC asociadas a su futuro profesional.

\begin{tabular}{cccccc}
\hline & Nula & Casi nada & Muy poco & Suficiente & Muchísimo \\
\hline $\begin{array}{c}\text { Programas específicos de su campo profesional (planificación, } \\
\text { evaluación, etc.) }\end{array}$ & 0,0 & 37,8 & 52,8 & 8,7 & 0,0 \\
$\quad \begin{array}{c}\text { Plataformas virtuales para formarse (plataformas de } \\
\text { enseñanza: campus virtual, moodle) }\end{array}$ & 0,0 & 33,1 & 59,1 & 5,5 & 0,0 \\
$\quad$ Pizarra electrónica o digital & 0,0 & 83,5 & 13,4 & 0,0 & 0,0 \\
\hline
\end{tabular}

Fuente: tabla elaborada por los autores para el presente estudio

Tabla 11- Correlación entre la frecuencia de uso y la percepción de dominio de herramientas TIC asociadas a su futuro profesional.

\begin{tabular}{ccccc}
\hline & \multicolumn{3}{c}{ Percepción de dominio } \\
\hline Frecuencia de uso & 32 & $\vdots$ & 39 & 44 \\
\hline Programas específicos de su campo profesional (planificación, evaluación, etc.) & $0,68^{*}$ & 0,05 & $0,18^{*}$ \\
Plataformas virtuales para formarse (plataformas de enseñanza: campus virtual, moodle) & $\vdots$ & $\vdots$ & $0,65^{*}$ & 0,03 \\
Pizarra electrónica o digital & $\vdots$ & $\vdots$ & & $0,55^{*}$ \\
\hline
\end{tabular}

Fuente: tabla elaborada por los autores para el presente estudio 
Situación similar ocurre con la frecuencia de uso y percepción de dominio de plataformas virtuales para formarse $(r=0,65, p$-value $<0,05)$ y la frecuencia de uso de la pizarra digital con su percepción de dominio $(r=0,55$, $p$-value $<0,05)$. No obstante lo anterior, existe un correlación directa entre la frecuencia de uso de programas específicos de su campo profesional con la percepción de dominio de la pizarra digital $(r=0,18, p$-value $<0,05)$.

\section{Discusión y conclusiones}

El estudio pretende obtener antecedentes a partir de los mismos actores involucrados, específicamente, la percepción de los estudiantes, para revisar los procesos de formación así como los currículos de las carreras de tipo pedagógicas.

Bajo este propósito, el estudio trabajó con las categorías de alfabetización tecnológica y alfabetización pedagógica. Por ello, operativamente se indagó sobre qué tan familiarizados están los estudiantes en formación inicial docente con las nuevas tecnologías, específicamente, en cuanto al acceso y uso de ellas; también sobre su autopercepción del dominio de la misma $y$, finalmente, sobre qué tan preparados se sienten para usar dichas tecnologías en su futuro desempeño profesional.

Si bien el tipo de estudio nos impide generalizar los resultados, al revisar lo datos podemos inferir los siguientes temas-problemas, a partir de los cuales se cuenta con la expectativa de generar un fructífero debate en virtud del mejoramiento de la formación inicial docente en lo referido a las competencias TIC, especialmente para el currículo y la acción didáctica.

\section{Alfabetización tecnológica versus alfabetización pedagógica en TIC}

Una de las principales conclusiones que se infiere de los datos, es el acceso generalizado a los recursos TIC que poseen los estudiantes, especialmente referido a la tenencia de computadores, ya sean fijos o portátiles (84,3\%), celulares $(99,2 \%)$ y conectividad a internet $(88,2 \%)$, lo que resulta relevante considerando que la muestra bajo estudio se caracteriza por pertenecer a los tres quintiles más bajos de la población del país. Sin embargo, ello es coherente con la última encuesta realizada por Pew Research Center (2014) sobre acceso a internet y tecnologías móviles en veinticuatro países clasificados como emergentes. En éste se destaca que en Chile, nueve de cada diez personas poseen un celular (siendo el tercer país con mayor acceso, sólo por debajo de Jordania y China). Así también, el país se ubica entre los tres primeros lugares de los veinticuatro países de la muestra, con alto porcentaje de acceso y uso de internet.

La misma encuesta señala que el tipo de uso de los celulares es variado, aunque mayoritariamente se usa para enviar mensajes de textos, fotografía (especialmente en los usuarios de Venezuela y Chile). Por su parte, acerca del acceso internet, los encuestados manifiestan que su uso mayoritario es para acceder a redes sociales y comunicarse con familiares y amigos, con un promedio de 96\% de preferencias.

Coherentemente con lo anterior, los datos del presente estudio muestran que la frecuencia y tipo de uso que los estudiantes dan a los computadores, internet y celulares, es para realizar trabajos (tareas) o comunicarse con familiares o entre ellos, con una frecuencia de uso que va desde mínimo cuatro a máximo los siete días a la semana.

Por todo lo anterior, se puede asumir que los estudiantes de Pedagogía son conocedores de las TIC siendo sujetos nativos en el acceso $\mathrm{y}$, por ende, en el uso de estas tecnologías de la información y comunicación, y por lo mismo, "piensan y procesan información de manera fundamentalmente distinta a sus antecesores. $\mathrm{Su}$ lengua nativa es el idioma digital de los computadores, los videojuegos e Internet" (PRENSKY, 2001 apud MARTÍNEZ, 2010, p. 63). 
No obstante lo anterior, no podemos inferir lo mismo en relación a dicha alfabetización y su uso potencial en su habitus profesional, ya que si bien los estudiantes se encuentran familiarizados en el uso de las TIC, no las suelen usar en el quehacer pedagógico o futuro quehacer profesional.

Al consultar por programas ligados al ejercicio futuro de la profesión, los estudiantes expresan que su dominio o conocimiento es escaso. Así pues, la opinión acerca de si dominan programas específicos para evaluar o planificar clases, las frecuencias en nulo o casi nada de dominio van desde $87,1 \%$ a $96,4 \%$. Situación similar ocurre sobre las plataformas de enseñanza, donde las frecuencias van desde $86,8 \%$ a $100 \%$ de dominio nulo o casi nada $y$, sobre el dominio de pizarra electrónica o digital, estos valores fluctúan ente un 66,7\% a 92,9\%.

$\mathrm{Si}$ bien existen algunas diferencias porcentuales en algunas carreras, podemos afirmar que, a nivel global, los estudiantes de Pedagogía manifiestan un escaso dominio de programas específicos para la profesión, con valores que fluctúan desde un 70\% hasta un 100\% de casi nada o nulo; dependiendo del programa.

Pon tanto, y siguiendo la argumentación anterior, podemos inferir, que los estudiantes encuestados si bien acceden y usufructúan de las TIC, desconocen cómo incorporarlas en su futuro profesional, ya sea porque no conocen ni usan programas específicos para el campo de la educación o bien, no logran vislumbrar la aplicación de aquellos que conocen y usan frecuentemente en sus futuras prácticas pedagógicas (ADELL, 2006; SANDOVAL; RODRÍGUEZ; MALDONADO, 2011).

En síntesis, podemos concluir a modo de hipótesis, que, en general, los estudiantes de Pedagogía mayoritariamente poseen una alfabetización tecnológica producto de la práctica social de la cultura digital que los rodea (SILVA, 2012); sin embargo, dicha alfabetización no les alcanza para ser alfabetizados pedagógicamente. Lo anterior cobra relevancia pues, tal como lo señala Peñaherrera, el uso de las TIC en el proceso enseñanza aprendizaje no depende "sólo de la tecnología sino también de las capacidades, actitudes y creencias pedagógicas de los profesores" (2012, p. 5).

\section{Alfabetización pedagógica en TIC y formación inicial docente}

Tal como se señaló anteriormente, si bien los estudiantes de Pedagogía poseen alta alfabetización tecnológica, no sucede lo mismo en su uso pedagógico. Por lo mismo, la formación inicial de los docentes debería centrar sus esfuerzos en la alfabetización pedagógica de las TIC para su adecuado uso en el sistema escolar y su aplicación en el proceso de enseñanza y aprendizaje.

Entonces debería estar en un ámbito diferente al de alfabetizar digitalmente a los estudiantes de pedagogía durante su formación, focalizándose en cómo alfabetizar pedagógicamente a los estudiantes para el uso de las TIC en sus prácticas o quehacer profesional futuro. Sin duda que el punto de partida son sus propias prácticas de alfabetización tecnológica, pero el desafío es cómo transferir este dominio a un contexto de prácticas pedagógicas, es decir, prácticas que se enmarcan en un espacio denominado aula, en un contexto social específico de escolarización, en función de un marco curricular escolar, con la finalidad de transmitir ciertos códigos sociales y culturales, mediante el empleo de diferentes medios y recursos (entre ellos los tecnológicos o TIC) para lograr un aprendizaje, habilidad o conocimiento por parte del educando.

En otras palabras, los futuros docentes deben aprender de forma práctica acerca del uso de la tecnología y ampliarlo con prácticas pedagógicas durante toda su formación, abarcando experiencia práctica y desarrollo profesional (SILVA, 2012). En otras palabras, se debería dar menos tiempo a la enseñanza de competencias tecnológicas, ya que son nativos y poseen una alfabetización digital y, por lo mismo, se debería focalizar el esfuerzo 
en las implicancias pedagógicas de dichas herramientas: cómo se integra al currículo, a los procesos didácticos y al proceso de enseñanza y aprendizaje que deberán conducir en su futuro profesional (COLL, 2004; SUÁREZ et al, 2010).

\section{Referencias}

ADELL, Jordi. Una odisea en la escuela. Cuadernos de Pedagogía, Barcelona, n. 363, p. 42-47, 2006.

CHILE. Ministerio de Educación. Enlaces: competencia y estándares TIC para la profesión docente. Santiago de Chile: Ministerio de Educación, 2011. Disponible en: <http://www.enlaces.cl/libros/docentes/index.html>. Acceso: 20 sept. 2013.

CHILE. Ministerio de Educación. Enlaces: innovación y calidad en la era digital: 20 años impulsando el uso de las TIC en educación. Santiago de Chile: Ministerio de Educación, 2012. Disponible en: <http://www.enlaces.cl/tp_enlaces/portales/tpe76eb4809f44/ uploadlmg/File/PDF/publicaciones/mem2013_baja.pdf>. Acceso: 20 sept. 2013.

COLL, César. Lectura y alfabetismo en la sociedad de la información. Revista Sobre la Sociedad del Conocimiento: UOCPapers, Barcelona, n. 1, p. 1-10, 2005. Disponible en: <http://www.uoc.edu/uocpapers/1/dt/esp/coll.pdf>. Acceso: 15 abr. 2014.

COLL, César. Psicología de la educación y prácticas educativas mediadas por las tecnologías de la información y la comunicación. Una mirada constructivista. Revista Electrónica Sinéctica, Jalisco, n. 25, p. 1-24, 2004. Disponible en: <http://www.sinectica. iteso.mx/assets/files/articulos/25_psicologia_de_la_educacion_y_practicas_educativas_mediadas_por_las_tecnologias_de_ la_informacion_y_la_comunicacionuna_mirada_constructivista.pdf>. Acceso: 12 abr. 2016.

COOK, Thomas; REICHARDT, Charles. Métodos cualitativos y cuantitativos en investigación evaluativa. Madrid: Morata, 1986.

CUADRADO, Isabel. Estado de necesidades, desarrollo y evaluación en formación de profesorado en TICS: extremadura. Revista Avance en Supervisión Educativa, Madrid, n. 8, p. 1-13, 2008. Disponible en: <http://www.adide.org/revista/index.php/ase/ article/view/322>. Acceso: 25 jun. 2016.

INE - Instituto Nacional de Estadística. Encuesta sobre equipamiento y uso de Tecnologías de Información y Comunicación en los Hogares (TIC-H). Madrid: INE, 2005. Disponible en: <http://www.ine.es/metodologia/t25/t25p450_tich_cues_05.pdf>. Acceso: 24 sept. 2013.

LATORRE, Antonio; DEL RINCÓN, Delio; ARNAL, Justo. Bases metodológicas de la investigación educativa. Barcelona: Hurtado, 1997.

MARTíNEZ, Hugo. La integración de las TIC en instituciones educativas. In: CARNEIRO, Roberto et al. (Ed.). Los desafíos de las TIC para el cambio educativo. Madrid: OEl: Fundación Santillana, 2010. p. 61-70.

PEIRANO, Claudia; DOMíNGUEZ, María Paz. Competencia en TIC: el mayor desafío para la evaluación y el entrenamiento docente en Chile. Revista Iberoamericana de Evaluación Educativa, Madrid, v. 1, n. 2, p. 107-124, 2008.

PEÑAHERRERA, Mónica. Uso de TIC en escuelas públicas de Ecuador: análisis, reflexiones y valoraciones. Revista Electrónica de Tecnología Educativa, Palma de Mallorca, n. 40, p. 1-16, 2012. Disponible en: <http://www.edutec.es/revista/index.php/ edutec-e/article/view/364>. Acceso: 20 jun. 2016.

PERAZZO, Mónica Isabel. La ruta de la alfabetización digital en la educación superior: una trama de subjetividades y prácticas. Revista de Universidad y Sociedad del Conocimiento, Cataluña, v. 5. n. 1, p. 1-10, 2008. Disponible en: <http://www.uoc.edu/ rusc/5/1/dt/esp/perazzo.pdf>. Acceso: 30 my. 2014.

Pew Research Center. Emerging nations embrace internet, mobile technology: cell phones nearly ubiquitous in many countries. Washingyon, DC: [s. n.], 2014. Disponible en: <http://www.pewglobal.org/files/2014/02/Pew-Research-Center-Global-AttitudesProject-Technology-Report-FINAL-February-13-20146.pdf>. Acceso: 30 my. 2014.

RIOSECO, Marcelo. Software libre como herramienta de producción científica en el ámbito educativo: el caso de Chile. Revista Latinoamericana de Tecnología Educativa, Cáceres v. 7, n. 2, p. 91-103, 2008. 
RODRÍGUEZ, José. Las alfabetizaciones digitales. Bordón, Madrid, v. 56, n. 3-4, p. 431-441, 2004.

SANDOVAL, Pedro; RODRÍGUEZ, Francisco; MALDONADO, Ana. Competencias TIC en la formación inicial docente: estudio descriptivo para la toma de decisiones en el currículum. Reflexão e Ação, Santa Cruz do Sul, v. 19, n. 1, p. 271-295, 2011.

SILVA, Juan. Estándares TIC para la formación inicial docente: una política pública en el contexto chileno. Archivos Analíticos de Políticas Educativas, Arizona v. 20, n. 7, p 1-35, 2012. Disponible en: <http://epaa.asu.edu/ojs/article/view/962>. Acceso: 30 ago. 2014.

SUÁREZ, Jesús et al. Las competencias en TIC del profesorado y su relación con el uso de los recursos tecnológicos. Archivos Analíticos de Políticas Educativas, Arizona, v. 18, n. 10, p. 1-28. 2010. Disponible en: <http://epaa.asu.edu/ojs/article/ view/755>. Acceso: 30 ago. 2014.

TELLO, Julio; AGUADED, José Ignacio. Desarrollo profesional docente ante los nuevos retos de la tecnología de la información y la comunicación en los centros educativos. Pixel-Bit, Sevilla, n. 34, p31-47, 2009. Disponible en: <http://rabida.uhu.es/dspace/ bitstream/handle/10272/6286/Desarrollo_profesional_docente.pdf?sequence=2>. Acceso: 30 ago. 2014 .

VAILLANT, Denise. Programa TIC y Educación Básica: integración de TIC en los sistemas de formación docente inicial y continua para la educación básica en América Latina. Buenos Aires: Fondo de las Naciones Unidas para la Infancia (UNICEF), 2013.

Recibido en: 28.09.15

Aprobado en:16.02.16

Pedro Sandoval Rubilar es doctor en ciencias de la educación (2003), académico en la Universidad del Bío-Bío desde el 2008 y coordinador Grupo de Investigación en Educación. Universidad del Bío-Bío.

Francisco Rodriguez Alveal es magíster en bioestadística (1996), académico en la Universidad del Bío-Bío desde el 2008 y miembro del Grupo de Investigación en Educación.

Ana Carolina Maldonado Fuentes es magíster en ciencias de la educación (1999), académica en la Universidad del Bío-Bío desde 2008 y miembro del Grupo de Investigación en Educación. 